\title{
Asteroseismology of the Kepler V777 Herculis variable white dwarf with fully evolutionary models
}

\author{
A. H. Córsico ${ }^{1,2}$, L. G. Althaus ${ }^{1,2}$, M. M. Miller Bertolami ${ }^{1,2}$, and A. Bischoff-Kim ${ }^{3}$ \\ ${ }^{1}$ Facultad de Ciencias Astronómicas y Geofísicas, Universidad Nacional de La Plata, Paseo del Bosque s/n, 1900 La Plata, Argentina \\ e-mail: [acorsico; althaus;miller]@fcaglp.unlp.edu.ar \\ 2 CONICET, Argentina \\ 3 Chemistry, Physics and Astronomy Department, Georgia College \& State University, Milledgeville, GA 31061, USA \\ e-mail: agnes.kim@gcsu.edu
}

Received 23 December 2011 / Accepted 13 March 2012

\section{ABSTRACT}

\begin{abstract}
Context. DBV stars are pulsating white dwarfs with atmospheres rich in He. Asteroseismology of DBV stars can provide valuable clues about the origin, structure and evolution of hydrogen-deficient white dwarfs, and may allow to study neutrino and axion physics. Recently, a new DBV star, KIC 8626021, has been discovered in the field of the Kepler spacecraft. It is expected that further monitoring of this star in the next years will enable astronomers to determine its detailed asteroseismic profile.

Aims. We perform an asteroseismological analysis of KIC 8626021 on the basis of fully evolutionary DB white-dwarf models.

Methods. We employ a complete set of evolutionary DB white-dwarf structures covering a wide range of effective temperatures and stellar masses. They have been obtained on the basis of a complete treatment of the evolutionary history of progenitors stars. We compute $g$-mode adiabatic pulsation periods for this set of models and compare them with the pulsation properties exhibited by KIC 8626021.

Results. On the basis of the mean period spacing of the star, we found that the stellar mass should be substantially larger than spectroscopy indicates. From period-to-period fits we found an asteroseismological model characterized by an effective temperature much higher than the spectroscopic estimate.

Conclusions. In agreement with a recent asteroseismological analysis of this star by other authors, we conclude that KIC 8626021 is located near the blue edge of the DBV instability strip, contrarily to spectroscopic predictions. We also conclude that the mass of KIC 8626021 should be substantially larger than thought.
\end{abstract}

Key words. stars: interiors - stars: oscillations - asteroseismology - white dwarfs - stars: evolution

\section{Introduction}

V777 Her (or DBV) stars are $g$-mode variable white dwarfs with He-rich atmospheres (DB) and effective temperatures in the range $21500 \lesssim T_{\text {eff }} \lesssim 29000 \mathrm{~K}$ that pulsate with periods between 100 and $1100 \mathrm{~s}$ (Winget \& Kepler 2008; Althaus et al. 2010). They are the hotter cousins of the ZZ Ceti (or DAV) stars, which are pulsating $\mathrm{H}$-rich atmosphere white dwarfs that define an instability strip centered at $T_{\mathrm{eff}} \approx 12000 \mathrm{~K}$. As an excellent demonstration of the validity of the stellar pulsation theory, the existence of the DBV class of compact pulsators was predicted on theoretical grounds (Winget et al. 1982a) before it were confirmed observationally (Winget et al. 1982b). Pulsations in V777 Her are thought to be driven by a combination of the $\kappa-\gamma$ mechanism acting in the He partial ionization zone - and thus setting the blue edge of the DBV instability strip (Winget et al. 1983; Bradley \& Winget 1994; Córsico et al. 2009), and the "convective driving" mechanism (Brickhill 1991; Goldreich \& Wu 1999) which is thought to be dominant once the outer convection zone has deepened enough.

White-dwarf asteroseismology - the comparison between the pulsation periods of white dwarfs and the periods computed for appropriate theoretical models - allows us to infer details of the origin, internal structure and evolution of white dwarfs. In particular, estimates of the stellar mass, $\mathrm{He}$ and $\mathrm{H}$ layer mass, core composition, magnetic field, rotation rate, seismological distance, and properties of the outer convection zone can be inferred from the observed pulsations of DAV and DBV stars (Winget \& Kepler 2008; Althaus et al. 2010). Finally, an eventual measurement of the temporal changes in the observed stable periods of DBVs could allow to study neutrino emission (Winget et al. 2004) and also axion emission (see Isern et al. 2010, and references therein).

Unlike the ZZ Ceti variables, that constitute the most numerous class of pulsating white dwarfs (about 150 objects are known today; Castanheira et al. 2010), V777 Her are quite rare and difficult to find, and until recently, only 20 stars of this class were known (Beauchamp et al. 1999; Nitta et al. 2009; Kilkenny et al. 2009). The list is now a bit enlarged with KIC 8626021 $\left(=\right.$ GALEX J192904.6+444708, $T_{\text {eff }}=24950 \pm 750 \mathrm{~K}$ and $\log g=7.91 \pm 0.07 \mathrm{dex}$ ), a very recently discovered DBV star located in the field of view of the Kepler mission by Østensen et al. (2011, hereinafter ØEA11) ${ }^{1}$. This star exhibits at least five periodicities in the range 197-376 s and amplitudes between 1 and $5.2 \mathrm{mma}$, with the three strongest modes showing a triplet structure due possibly to rotation. It is expected that additional modes with higher radial orders (longer periods) will be detected in future runs of Kepler, thus increasing the potential of asteroseismology to infer its interior structure. Given

${ }^{1}$ Even more recently, Hermes et al. (2011) have reported the discovery of the first DAV star in the Kepler mission field. 
the values of $T_{\text {eff }}$ and $\log g$ quoted above and the DB models of Althaus et al. (2009a), KIC 8626021 should have a mass of $M_{*}=0.56 \pm 0.03 M_{\odot}$.

Motivated by the exciting discovery of the first pulsating white dwarf in the Kepler field of view, we present an asteroseismological analysis of KIC 8626021 on the basis of the DB white-dwarf models presented in Althaus et al. (2009a). This grid of evolutionary models was computed for a wide range of stellar masses on the basis of a complete treatment of the evolutionary history of progenitors stars, including those stages relevant for the chemical profiles of the white dwarf, such as the core $\mathrm{H}$ and $\mathrm{He}$ burning phases, the thermally pulsing asymptotic giant branch phase, and the born-again episode that is responsible for the $\mathrm{H}$ deficiency.

By considering the mean period spacing exhibited by the star, we found that KIC 8626021 should be substantially more massive than spectroscopy indicates. We also found that period to period fits favour an asteroseismological solution characterized by an effective temperature much higher than the spectroscopic estimate.

While writing this paper, Bischoff-Kim \& Østensen (2011) (hereinafter BKØ11) announced the results of their own independent asteroseismological analysis on KIC 8626021. They found that this star is actually a hot DBV. Using entirely different models and methods, we arrive at the same conclusion. The fundamental difference between our approach and that of BKØ11 is that we run evolutionary models starting on the Zero Age Main Sequence, accounting for nuclear burning, time dependent diffusion of the elements and other physical processes that take place in the course of the evolution of a star. BKØ11 perform "fast white dwarf asteroseismology", where they guess and parameterize the internal chemical composition profiles and build static models. That method allows them a fuller exploration of parameter space. For KIC 8626021, they find very good fits (hotter than what the preliminary spectroscopy suggests) but also conclude that their internal Oxygen composition profiles are in poor agreement with stellar evolution calculations. They suggest further studies to see what the physical parameters of models that agree with stellar evolution calculations would be. In essence, this is the kind of study we present here. While our best fit models are evidently different, we concur with the conclusion that KIC 8626021 is hotter (and more massive) than suggested by the initial spectroscopic study. The conclusion that this star is residing at the blue edge of the DBV instability strip appears to be robust, and calls for the necessity of a new and improved spectroscopic determination of its effective temperature.

In Sect. 2 we present some details about our models and methods. In Sect. 3, we use the average period spacing of KIC 8626021's period spectrum to draw some conclusions about its mass and effective temperature. We contrast our models to the grid of models computed by BKØ11. In Sect. 4, we present our best fit models. Again, we contrast our results with those of BKØ11. In Sect. 5 we discuss the discrepancies between the results coming from spectroscopy and from asteroseismology, and the differences between our asteroseismological results and those of BKØ11. We conclude in Sect. 6.

\section{Modeling}

\subsection{Numerical codes and asteroseismological approach}

The evolutionary DB white dwarf models employed in this work were presented in Althaus et al. (2009a) and we refer to that paper for details. Briefly, the models were computed with the LPCODE stellar evolutionary code we employed in our previous studies on the formation of PG 1159 and extreme horizontal branch stars (Althaus et al. 2005; Miller Bertolami \& Althaus 2006; Miller Bertolami et al. 2008), hot DQ white dwarfs (Althaus et al. 2009b) as well as the formation and evolution of He-core white dwarfs with high metallicity progenitors (Althaus et al. 2009c). The LPCODE evolutionary code considers a simultaneous treatment of non-instantaneous mixing and burning of elements, which is of primary importance for the calculation of chemical abundance changes during the short-lived evolutionary stages characteristic of unstable burning episodes, like the born-again stage, from which our starting H-deficient white dwarf configurations are derived. Nuclear reaction rates are from Caughlan \& Fowler (1988) and Angulo et al. (1999). The ${ }^{12} \mathrm{C}(\alpha, \gamma){ }^{16} \mathrm{O}$ reaction rate was taken from Angulo et al. (1999), which is about twice as large as that of Caughlan \& Fowler (1988). A moderate, diffusive overshooting in the core and in the envelope is allowed during pre-white-dwarf evolution. For the white dwarf regime, we considered the following main physical ingredients. Neutrino emission rates for pair, photo, and bremsstrahlung processes are those of Itoh et al. (1996). For plasma processes, we use the treatment presented in Haft et al. (1994). Radiative opacities are those of the OPAL project (Iglesias \& Rogers 1996), including C- and O-rich composition. We adopted the conductive opacities of Cassisi et al. (2007). This prescription covers the whole regime where electron conduction is relevant. For the high density regime, we used the equation of state of Segretain et al. (1994), which accounts for all the important contributions for both the liquid and solid phases. For the low-density regime, we used an updated version of the equation of state of Magni \& Mazzitelli (1979). Convection was treated in the formalism of the mixing-length theory as given by the ML2 parameterization (Tassoul et al. 1990). All of our white dwarf sequences were computed in a consistent way with the evolution of the chemical abundance distribution caused by element diffusion along the whole cooling phase. In particular, we considered gravitational settling and chemical diffusion of ${ }^{4} \mathrm{He},{ }^{12} \mathrm{C},{ }^{13} \mathrm{C}$, ${ }^{14} \mathrm{~N}$, and ${ }^{16} \mathrm{O}$.

The adiabatic pulsation periods employed in the present study were assessed with the help of the pulsational code described in Córsico \& Althaus (2006). The prescription we follow to assess the run of the Brunt-Väisälä frequency $(N)$ is the socalled "Ledoux Modified" treatment - see Tassoul et al. (1990) - appropriately generalized to include the effects of having three nuclear species $(\mathrm{O}, \mathrm{C}$, and $\mathrm{He}$ ) varying in abundance.

Our asteroseismological approach basically consists in the employment of detailed white dwarf models characterized by very accurate physical ingredients. These models are obtained by computing the complete evolution of the progenitor stars. We have applied successfully this approach to the hot DOVs or GW Vir stars (see Córsico et al. 2007a,b, 2008, 2009) and recently to an ensemble of bright ZZ Ceti stars (Romero et al. 2012). Since the final chemical stratification of white dwarfs is fixed in prior stages of their evolution, the evolutionary history of progenitor stars is of utmost importance in the context of white dwarf asteroseismology. Our asteroseismological approach, while being physically sound, is by far more computationally demanding than other approaches in which simplified models are used. As a result, our approach severely limits the exploration of the parameter space of the models. Indeed, for the case of DB white dwarfs, we have only two parameters which we are able to vary in a consistent way: the stellar mass $\left(M_{*}\right)$ and the effective temperature $\left(T_{\text {eff }}\right)$. Instead, the content of $\mathrm{He}\left(M_{\mathrm{He}}\right)$, the shape of the $\mathrm{C}-\mathrm{O}$ chemical structure at the core (including the precise 
proportions of central $\mathrm{O}$ and $\mathrm{C}$ ), and the thickness of the chemical transition regions are fixed by the evolutionary history of progenitor stars.

\subsection{DB white dwarf evolutionary models}

The initial models for our DB white dwarf sequences correspond to realistic PG 1159 stellar configurations derived from the full evolutionary calculations of their progenitor stars (Miller Bertolami \& Althaus 2006). All the sequences were computed from the ZAMS through the thermally-pulsating and mass-loss phases on the AGB and finally to the born-again stage where the remaining $\mathrm{H}$ is violently burnt. After the born again episode, the $\mathrm{H}$-deficient, quiescent He-burning remnants evolve at constant luminosity to the domain of PG1159 stars with a surface chemical composition rich in $\mathrm{He}, \mathrm{C}$ and $\mathrm{O}$ (Miller Bertolami \& Althaus 2006). This new generation of PG 1159 evolutionary models has succeed in explaining both the spread in surface chemical composition observed in most PG 1159 stars and the location of the GW Vir instability strip in the $\log T_{\text {eff }}-\log g$ plane (Córsico et al. 2006). Also, these PG 1159 models have been employed in detailed asteroseismological studies of six pulsating PG1159 stars (Córsico et al. 2007a,b, 2008, 2009).

Specifically, we considered nine DB white dwarf sequences with stellar masses of: $0.515,0.530,0.542,0.565,0.584,0.609$, $0.664,0.741$, and $0.870 M_{\odot}$. These DB sequences are characterized by the maximum He-rich envelope that can be left by prior evolution if we assume that they are the result of a born-again episode. The value of envelope mass ranges from $M_{\mathrm{He}} / M_{*} \sim 2 \times 10^{-2}\left(M_{*}=0.515 M_{\odot}\right)$ to $M_{\mathrm{He}} / M_{*} \sim 1 \times 10^{-3}$ $\left(M_{*}=0.870 M_{\odot}\right)$. The complete set of DB white dwarf evolutionary sequences is displayed in Fig. 1 with different colors according to the value of the stellar mass. Also shown is the location of the known DBV stars with values of $T_{\text {eff }}$ and $\log g$ extracted from Beauchamp et al. (1999, for the case in which no traces of $\mathrm{H}$ are considered; black circles), from Nitta et al. (2009) for the nine DBV stars of the SDSS (black squares), and Kilkenny et al. (2009) (black triangle). The location of KIC 8626021 according to ØEA11 is highlighted with a red star symbol.

\subsection{A template model}

We briefly describe the main pulsation properties of our DB white dwarf models. We focus on a template DB model with $M_{*}=0.565 M_{\odot}$ and $T_{\text {eff }} \sim 28400 \mathrm{~K}$. In Fig. 2 we depict the internal chemical structure of such model (upper panel), where the abundance by mass of the main constituents $\left({ }^{4} \mathrm{He},{ }^{12} \mathrm{C}\right.$, and $\left.{ }^{16} \mathrm{O}\right)$ is shown in terms of the outer mass fraction $\left[-\log \left(1-M_{r} / M_{*}\right)\right]$. The chemical structure of our models consists of a $\mathrm{C} / \mathrm{O}$ core resulting from the core $\mathrm{He}$ burning of the previous evolution - shaped by processes of extra mixing like overshooting. The core is surrounded by a mantle rich in $\mathrm{He}, \mathrm{C}$, and $\mathrm{O}$ which is the remnant of the regions altered by the nucleosynthesis during the thermally pulsing asymptotic giant branch. Above this shell, there is a pure He mantle with a mass $M_{\mathrm{He}} / M_{*} \sim 6.7 \times 10^{-3}$, constructed by the action of gravitational settling that causes He to float to the surface and heavier species to sink. A double-layered structure of the He-rich envelope is clearly visible (Dehner \& Kawaler 1995; Gautschy \& Althaus 2002; Althaus \& Córsico 2004). In our models, the shape of the double-layered structure evolves with time (with appreciable changes even within the DBV instability strip) by virtue of time-dependent chemical diffusion processes (see Althaus \& Córsico 2004).

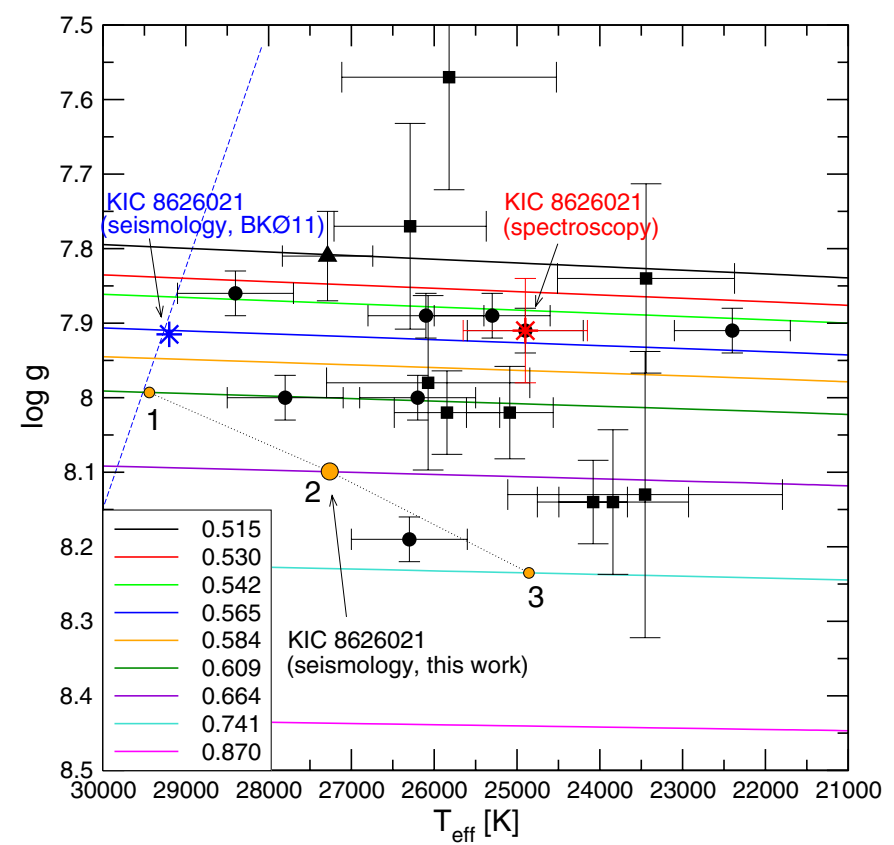

Fig. 1. The location of the known DBV stars on the $T_{\mathrm{eff}}-\log g$ plane. Also included are our DB white dwarf evolutionary tracks displayed with different colors according to the stellar mass. The location of KIC 8626021 according to spectroscopy is emphasized with a red star symbol, while the location of our asteroseismological models for this star is marked with orange circles connected with a dotted line. The largest circle corresponds to our best-fit model (model 2; see Sect. 4). For comparison, we include the location of the best-fit asteroseismological model of BKØ11 with a blue star symbol. The theoretical blue edge of the DBV instability strip corresponding to the version MLT2 ( $\alpha=1.25$ ) of the MLT theory of convection, as derived by Córsico et al. (2009), is depicted with a blue dashed line. Note: the DBV star EC 05221 (Kilkenny et al. 2009) is not included because at present there is no any estimation of $T_{\text {eff }}$ and $\log g$ available.

The lower panel of Fig. 2 displays the run of the two critical frequencies of nonradial stellar pulsations, that is, the Brunt-Väisälä frequency and the Lamb frequency $\left(L_{\ell}\right)$ for $\ell=1$. The precise shape of the Brunt-Väisälä frequency largely determines the properties of the $g$-mode period spectrum of the model. In particular, each chemical gradient in the model contributes locally to the value of $N$. The most notable features are the very peaked feature at the $\mathrm{C} / \mathrm{O}$ chemical transition $\left[-\log \left(1-M_{r} / M_{*}\right) \sim 0.3\right]$, and the bump at the He/C/O interface $\left[-\log \left(1-M_{r} / M_{*}\right) \sim 1.5\right]$. On the other hand, the $\mathrm{He} / \mathrm{C} / \mathrm{O}$ transition region at $-\log \left(1-M_{r} / M_{*}\right) \sim 5$ is very smooth and does not affect the pulsation spectrum much. For comparison, we also include in Fig. 2 the core chemical profiles and the BruntVäisälä frequency of the best fit model for KIC 8626021 found by BKØ11 (thin lines).

\section{Period spacing: the global information}

For $g$-modes with high radial order $k$ (long periods), the separation of consecutive periods $(|\Delta k|=1)$ becomes nearly constant at a value given by the asymptotic theory of nonradial stellar pulsations. Specifically, the asymptotic period spacing (Tassoul et al. $1990)$ is given by:

$\Delta \Pi_{\ell}^{\mathrm{a}}=\Pi_{0} / \sqrt{\ell(\ell+1)}$, 


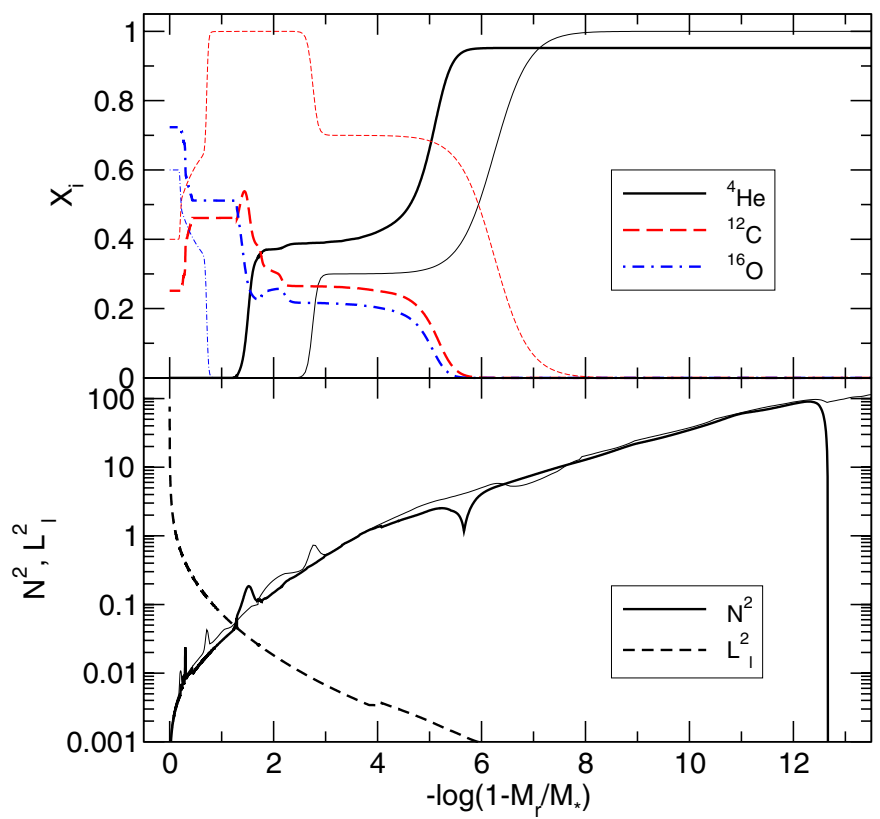

Fig. 2. The internal chemical structure (upper panel), and the squared Brunt-Vaïsälä and Lamb frequencies for $\ell=1$ (lower panel) corresponding to our template DB white dwarf model with a stellar mass $M_{*}=0.565 M_{\odot}$, an effective temperature $T_{\text {eff }} \sim 28400 \mathrm{~K}$, and a He envelope mass of $M_{\mathrm{He}} / M_{*} \sim 6.7 \times 10^{-3}$ (thick lines). For comparison, we show with thin lines the chemical profiles and the Brunt-Väisälä frequency of the best fit model for KIC 8626021 found by BKØ11.

where

$\Pi_{0}=2 \pi^{2}\left[\int_{r_{1}}^{r_{2}} \frac{N}{r} \mathrm{~d} r\right]^{-1}$.

This expression is rigorously valid for chemically homogeneous stars. In principle, one can compare the asymptotic period spacing computed from a grid of models with different masses and effective temperatures with the mean period spacing exhibited by the star, and then infer the value of the stellar mass. This method has been applied in numerous studies of pulsating PG 1159 stars (see, for instance, Córsico et al. 2007a,b, 2008, 2009, and references therein). For the method to be valid, the periods exhibited by the pulsating star must be associated with high order $g$-modes, that is, the star must be within the asymptotic regime of pulsations. KIC 8626021 exhibits pulsation periods associated to low order modes, with presumably $k \lesssim 10$. Furthermore, the interior of DB white dwarf stars are supposed to be chemically stratified and characterized by strong chemical gradients built up during the progenitor star life. So, the direct application of the asymptotic period spacing to infer the effective temperature and stellar mass of KIC 8626021 may appear somewhat questionable. However, the observed period spectrum is roughly evenly spaced, indicating that using asymptotic theory may not be an unreasonable thing to do. In order to compare our models to the period spacing of the observed pulsation spectrum, we calculate the average of the computed period spacings, using:

$\overline{\Delta \Pi}_{\text {the }}\left(M_{*}, T_{\text {eff }}\right)=\frac{1}{(n-1)} \sum_{k}^{n-1} \Delta \Pi_{k}$,

where $\Delta \Pi_{k}$ is the "forward" period spacing defined as $\Delta \Pi_{k}=$ $\Pi_{k+1}-\Pi_{k}$, and $n$ is the number of computed periods laying in the range of the observed periods. The theoretical period spacing of

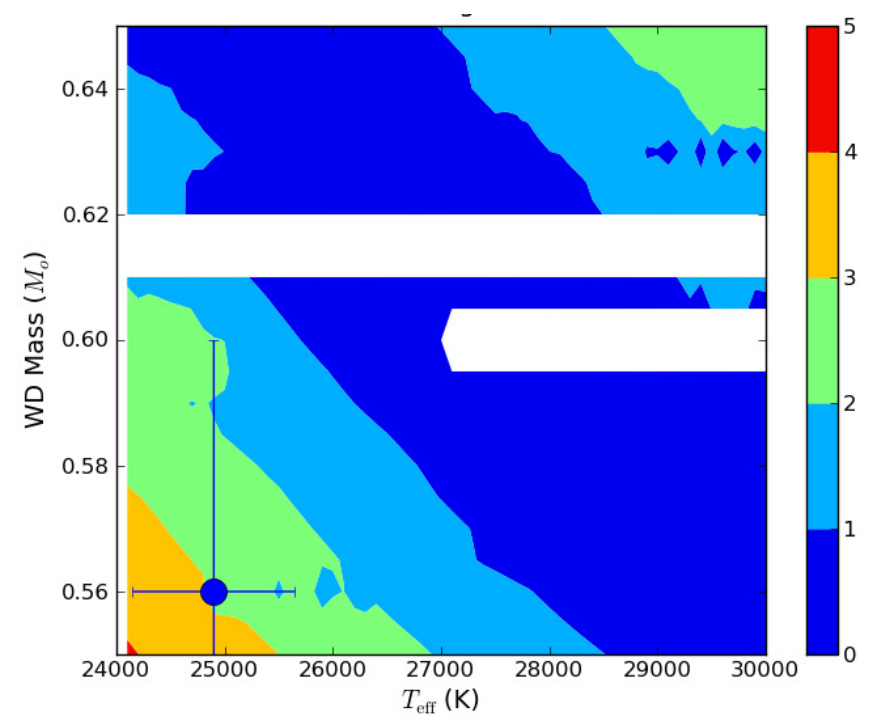

Fig. 3. The location of the models of BKØ11 with an average period spacing close to that of KIC 8626021 in the mass-temperature plane. The colour scale is related to the the difference between the observed average period spacing of the star and the period spacing computed with Eq. (3). The spectroscopic values for the mass and temperature of the star are also indicated with the point and error bars. It is obviously outside the region of the best fits in average period spacing. The white regions are regions where models failed to converge. They do not affect the results.

the models as computed through Eqs. (1) and (3) share the same general trends (that is, the same dependence on $M_{*}$ and $T_{\text {eff }}$ ), although $\Delta \Pi_{\ell}^{\mathrm{a}}$ is usually somewhat higher than $\overline{\Delta \Pi}_{\text {the }}$ provided that this last quantity is computed on a range of periods associated to low order modes (short periods). We estimate the observed mean period spacing of KIC 8626021 by means of a nonlinear leastsquares fit by considering the five periods observed in the star (first column of Table 2 ). We obtain $\overline{\Delta \Pi}_{\mathrm{obs}}=35.78 \pm 0.47 \mathrm{~s}$.

To check the validity of using the period spacing to study KIC 8626021, we use the grid of models computed by BKØ11 (400000 models). For the fixed internal chemical composition profiles depicted in Fig. 2 and for each model, we calculate the difference between the observed average period spacing of the star and the calculated period spacing (Eq. (3)). The result is presented in Fig. 3. A striking feature is the diagonal region where the spacings are close. This is a direct consequence of Eq. (2) and evidence that asymptotic theory applies at least in broad strokes. In Eq. (2), the dependence on the Brunt-Väisälä frequency is such that the asymptotic period spacing is greater when the mass and/or temperature of the model is higher. This dependence is apparent in Fig. 3. In order to keep a constant average period spacing (that of the star), one has to compensate lower temperatures with higher masses and vice versa.

In Fig. 4 we show the run of average of the computed period spacings (Eq. (3)) in terms of the effective temperature for all of our DB white-dwarf evolutionary sequences. The curves displayed in the plot are somewhat jagged. This is because the average of the computed period spacings is evaluated for a fixed period interval, and not for a fixed $k$-interval. As the star evolves towards lower effective temperatures, the periods generally increase with time. At a given $T_{\text {eff }}$, there are $n$ computed periods laying in the chosen period interval. Later, when the model has cooled enough, it is possible that the accumulated period drift nearly matches the period separation between 


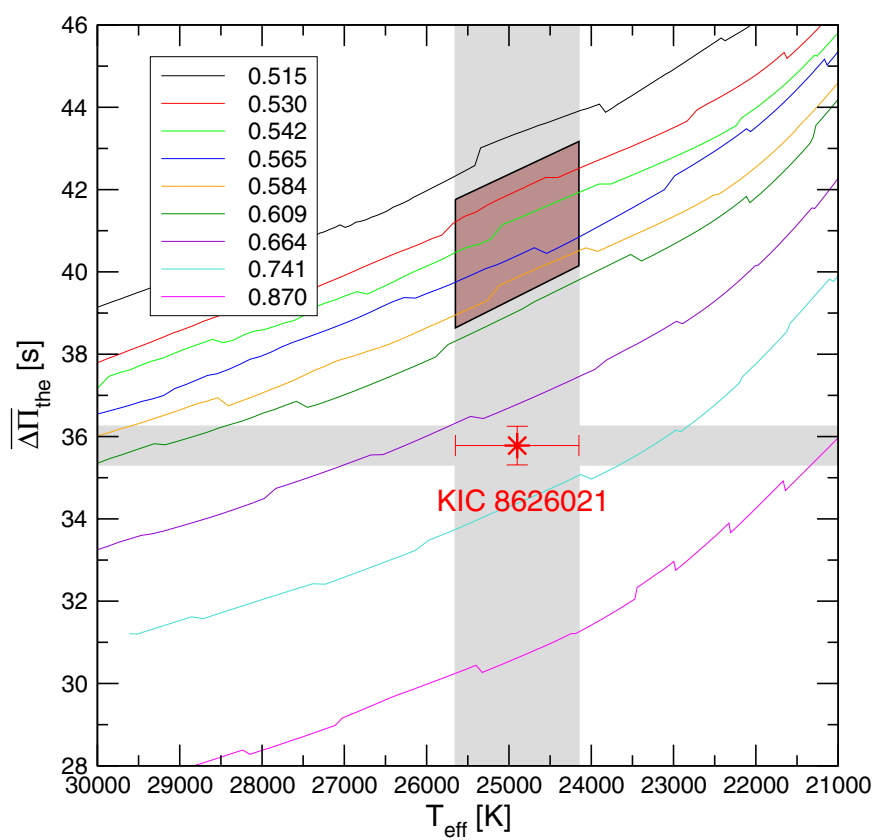

Fig. 4. The average of the computed period spacings corresponding to our DB white dwarf sequences with different stellar masses. The location of KIC 8626021 is shown with a red star symbol $\left(T_{\text {eff }}=\right.$ $24900 \pm 750 \mathrm{~K}, \overline{\Delta \Pi}_{\mathrm{obs}}=35.78 \pm 0.47 \mathrm{~s}$ ). By simple linear interpolation, we found that the mass of the star according to its period spacing is $M_{*}=0.696 \pm 0.031 M_{\odot}$. According to spectroscopy $(\log g=$ $7.91 \pm 0.07$; see Fig. 1), the period spacing of the star should be somewhere within the dark shaded area.

adjacent modes $(|\Delta k|=1)$. In these circumstances, the number of periods laying in the chosen (fixed) period interval is $n \pm 1$, and $\overline{\Delta \Pi}_{\text {the }}$ exhibits a little jump. In order to iron out the curves of $\overline{\Delta \Pi}_{\text {the }}$, in constructing Fig. 4 we have considered pulsation periods in a wider range of periods $\left(100 \lesssim \Pi_{k} \lesssim 1200 \mathrm{~s}\right)$ than that shown by KIC $8626021\left(195 \lesssim \Pi_{k} \lesssim 380 \mathrm{~s}\right)^{2}$. The location of KIC 8626021 is emphasized with a red star symbol. If we adopt the spectroscopic effective temperature of KIC 8626021 $\left(T_{\text {eff }}=24900 \pm 750 \mathrm{~K}\right.$; ØEA11) we infer a mass for the star according to its mean period spacing of $M_{*}=0.696 \pm 0.031 M_{\odot}$, which is $\sim 24 \%$ larger than the spectroscopic estimate $\left(M_{*}=\right.$ $\left.0.56 \pm 0.03 M_{\odot}\right)$. The dark shaded area in the figure shows the location of the star $\left(\overline{\Delta \Pi}_{\text {the }}, T_{\text {eff }}\right)$ according to the constraint given on gravity by spectroscopy $(\log g=7.91 \pm 0.07$; see Fig. 1$)$. The strong discrepancy between the predictions of spectroscopy and the period spacing estimator is vividly depicted in this figure. This difference between the spectroscopic and seismic masses is particularly relevant as, ultimately, both estimates of the stellar mass of KIC 8626021 are based on the same DB white dwarf evolutionary models.

This estimate of the stellar mass is based on the reliability of the spectroscopic determination of the effective temperature. In this sense, it would be quite interesting to have an estimate of the stellar mass devoid of any possible uncertainty from the spectroscopic analysis. If we relax for the moment the constraint imposed by the effective temperature, and look for the possible range of masses for the star to be within the DBV instability strip $\left(21500 \lesssim T_{\text {eff }} \lesssim 29000 \mathrm{~K}\right)$, we find that $0.60 \lesssim M_{*} / M_{\odot} \lesssim 0.87$.

\footnotetext{
${ }^{2}$ If we adopt a shorter range of periods, closer to the range of periods exhibited by KIC 8626021 (say 195-380 s), the curves we obtain are much more irregular and jumped, although the results in relation to KIC 8626021 do not appreciable change.
}

Confirming the findings of BKØ11 (see also Fig. 3), the rather low mass value predicted by spectroscopy $\left(M_{*} \sim 0.56 M_{\odot}\right)$ is excluded by the mean period spacing, unless the star is outside the instability strip ( $T_{\text {eff }} \gtrsim 32000 \mathrm{~K}$; see Fig. 4). This conclusion relies only on the measured mean period spacing, which is a quantity accurately measured, and on the average of the computed period spacing, a global quantity that is not affected by the precise shape of the chemical transition regions of the models. It is particularly worth noting that this result is independent of the mass of the He-rich envelope. Indeed, Tassoul et al. (1990) have shown that the asymptotic period spacing of DBV white dwarfs is particularly insensitive to the mass of the He-rich mantle, changing less than $1 \mathrm{~s}$ for a change of 10 orders of magnitude in the mass of the He-rich mantle (see their Fig. 42). Also, this conclusion is independent of the effective temperature estimation of the star. So, the result that KIC 8626021 is more massive and/or hotter than indicated by spectroscopy seems to be a robust finding ${ }^{3}$.

\section{Period fits: letting the pulsation periods to speak by themselves}

Another way to infer the stellar mass, effective temperature, and details of the internal structure of KIC 8626021 is through its individual pulsation periods, which are extracted from ØEA11 and are listed in the first column of Table 2. In this approach we seek a pulsation DB white dwarf model that best matches the pulsation periods of KIC 8626021 . We assume that all of the observed periods correspond to $\ell=1$ modes, to be consistent with the mean period spacing. The goodness of the match between the theoretical pulsation periods $\left(\Pi_{k}\right)$ and the observed individual periods $\left(\Pi_{\mathrm{obs}, i}\right)$ is measured by means of a quality function defined as:

$\chi^{2}\left(M_{*}, T_{\mathrm{eff}}\right)=\frac{1}{N} \sum_{i=1}^{N} \min \left[\left(\Pi_{\mathrm{obs}, i}-\Pi_{k}\right)^{2}\right]$,

where $N(=5)$ is the number of observed periods. The DB white dwarf model that shows the lowest value of $\chi^{2}$ is adopted as the "best-fit model" (see Córsico et al. 2007a,b, 2008, 2009; Romero et al. 2012). We evaluate the function $\chi^{2}\left(M_{*}, T_{\text {eff }}\right)$ for stellar masses of $0.515,0.530,0.542,0.565,0.584,0.609,0.664,0.741$, and $0.870 M_{\odot}$. For the effective temperature we employed a much more finer grid $\left(\Delta T_{\text {eff }}=10-30 \mathrm{~K}\right)$. The quality of our period fits is assessed by means of the average of the absolute period differences, $\bar{\delta}=\left(\sum_{i=1}^{N}\left|\delta_{i}\right|\right) / N$, where $\delta_{i}=\Pi_{\mathrm{obs}, i}-\Pi_{k}$, and by the root-mean-square residual, $\sigma=\sqrt{\left(\sum\left|\delta_{i}\right|^{2}\right) / N}=\sqrt{\chi^{2}}$.

The quantity $\left(\chi^{2}\right)^{-1}$ in terms of the effective temperature for different stellar masses is shown in Fig. 5 together with the spectroscopic effective temperature of KIC 8626021 (red line) and its uncertainties (gray strip). We found one strong maximum for a model with $M_{*}=0.664 M_{\odot}$ and $T_{\text {eff }}=27260 \mathrm{~K}$ (model 2$)$. Such a pronounced maximum in the inverse of $\chi^{2}$ implies an excellent agreement between the theoretical and observed periods. Notably, the effective temperature of this model is much higher (about $2400 \mathrm{~K}$ larger) than the spectroscopic effective temperature of KIC 8626021. Another maximum, albeit much less pronounced, is encountered for quite hotter and somewhat less massive model with $T_{\text {eff }}=29440$ and $M_{*}=0.609 M_{\odot}$

3 If we apply the method described above but employing the asymptotic period spacing $\Delta \Pi_{\ell}^{\mathrm{a}}$ (Eq. (1)), we obtain $M_{*} \sim 0.80 M_{\odot}$, and if we relax the constraint of the spectroscopic $T_{\text {eff }}$, we find that $0.66 \lesssim$ $M_{*} / M_{\odot} \lesssim 0.90$ for the star to be within the DBV instability strip. 
Table 1. Asteroseismological solutions for KIC 8626021.

\begin{tabular}{lcccccc}
\hline \hline Model & $\begin{array}{c}T_{\text {eff }} \\
{[\mathrm{K}]}\end{array}$ & $\begin{array}{c}\log g \\
{\left[\mathrm{~cm} \mathrm{~s}^{-2}\right]}\end{array}$ & $\begin{array}{c}M_{*} \\
{\left[M_{\odot}\right]}\end{array}$ & $\begin{array}{c}\bar{\delta} \\
{[\mathrm{s}]}\end{array}$ & $\begin{array}{c}\sigma \\
{[\mathrm{s}]}\end{array}$ & BIC \\
\hline 1 & 29441 & 7.993 & 0.609 & 2.314 & 2.721 & 1.15 \\
2 & 27263 & 8.099 & 0.664 & 1.582 & 1.934 & 0.85 \\
3 & 24856 & 8.235 & 0.741 & 3.334 & 3.685 & 1.41 \\
\hline
\end{tabular}

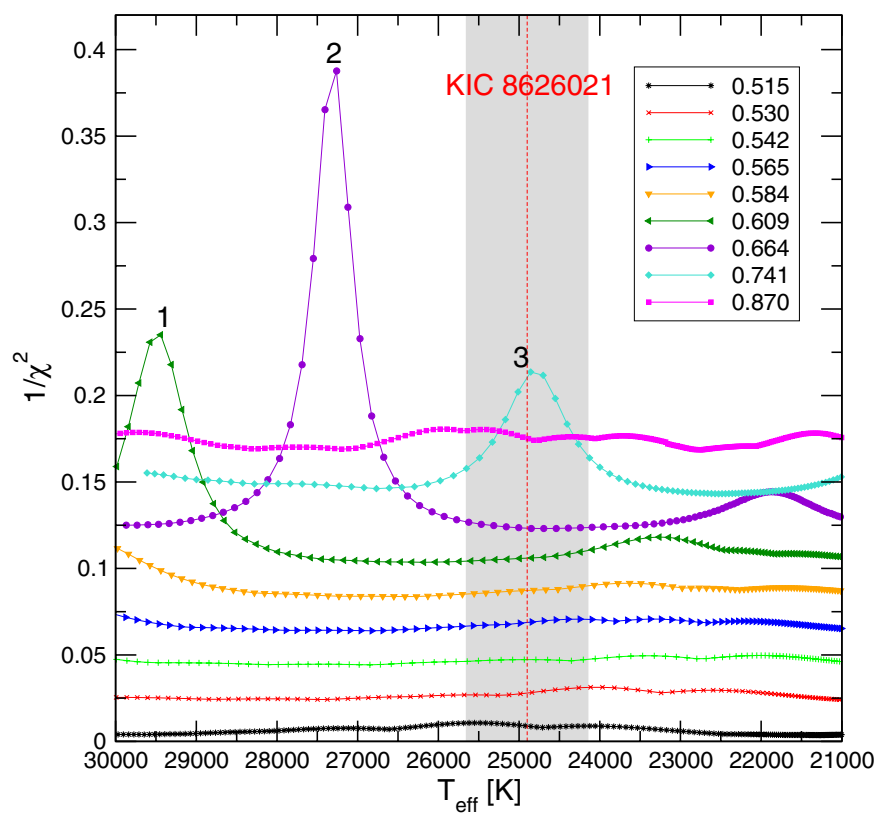

Fig. 5. The inverse of the quality function of the period fit in terms of the effective temperature. The vertical gray strip indicates the spectroscopic $T_{\text {eff }}$ and its uncertainties ( $\left.T_{\text {eff }}=24900 \pm 750 \mathrm{~K}\right)$. The curves have been arbitrarily shifted upward (with a step of 0.02). The strong maximum in $\left(\chi^{2}\right)^{-1}$ corresponds to the best-fit model (model 2 in Table 1), with $M_{*}=0.664 M_{\odot}$ and $T_{\mathrm{eff}}=27263 \mathrm{~K}$.

(model 1). According to its $T_{\text {eff }}$, this model is in excellent agreement with the asteroseismological solution found by BKØ11 $\left(T_{\text {eff }}=29200 \mathrm{~K}\right.$; see Table 3 below $)$. Finally, a third asteroseismological solution is found at a model with $M_{*}=0.741 M_{\odot}$ and $T_{\text {eff }}=24856$ (model 3). Interestingly, this model has exactly the spectroscopically inferred effective temperature of KIC 8626021, $T_{\text {eff }} \approx 24900 \mathrm{~K}$. In Table 1 we summarize the main characteristics of the three solutions we found in our analysis, that is, models 1, 2 and 3. Models 1 and 3 constitute acceptable asteroseismological solutions. However, because the agreement between observed and theoretical periods for these models are much poorer than for model 2 (see Cols. 5 and 6 of Table 1), we adopt this last model as the best-fit asteroseismological model of KIC 8626021 . One should bear in mind that the analysis of the mean period spacing suggests a preferred mass of $\sim 0.7 M_{\odot}$, between our $0.664 M_{\odot}$ and $0.741 M_{\odot}$ models. Thus a better fit, with a mass between $0.664 M_{\odot}$ and $0.741 M_{\odot}$, might had arisen if a thinner mass grid were available.

A detailed comparison of the observed $m=0$ periods in KIC 8626021 with the theoretical periods of the best-fit asteroseismological model is provided in Table 2. For this model, we obtain $\bar{\delta}=1.582 \mathrm{~s}$ and $\sigma=1.934 \mathrm{~s}$. The mean period spacing of our best fit model is $\overline{\Delta \Pi}=35.10 \pm 0.50 \mathrm{~s}$ (nonlinear leastsquares fit), in excellent agreement with the mean period spacing of $\mathrm{KIC} 8626021\left(\overline{\Delta \Pi}_{\mathrm{obs}}=35.78 \pm 0.47\right)$.
Table 2. Comparison between the observed periods of KIC 8626021 and the theoretical periods of the best-fit model (model 2 in Table 1).

\begin{tabular}{lcccccc}
\hline \hline $\begin{array}{l}\Pi_{\mathrm{obs}, i} \\
{[\mathrm{~s}]}\end{array}$ & $\begin{array}{c}A_{i} \\
{[\mathrm{mma}]}\end{array}$ & $\begin{array}{c}\Pi_{k} \\
{[\mathrm{~s}]}\end{array}$ & $\ell$ & $k$ & $\begin{array}{c}\delta_{i} \\
{[\mathrm{~s}]}\end{array}$ & $\begin{array}{c}\mathrm{d} \Pi_{k} / \mathrm{d} t \\
{\left[10^{-14} \mathrm{~s} / \mathrm{s}\right]}\end{array}$ \\
\hline 197.11 & 2.92 & 200.43 & 1 & 3 & -3.32 & 5.60 \\
232.02 & 5.21 & 233.69 & 1 & 4 & -1.67 & 8.66 \\
271.60 & 1.87 & 271.22 & 1 & 5 & 0.38 & 6.84 \\
303.56 & 1.29 & 301.42 & 1 & 6 & 2.14 & 9.91 \\
- & - & 339.69 & 1 & 7 & - & 6.67 \\
376.10 & 1.05 & 376.50 & 1 & 8 & -0.40 & 12.13 \\
\hline
\end{tabular}

Table 3. The main characteristics of KIC 8626021.

\begin{tabular}{lccc}
\hline \hline Quantity & $\varnothing$ EA11 & BKØ11 & This work \\
\hline$T_{\text {eff }}[\mathrm{K}]$ & $24950 \pm 750$ & 29200 & 27263 \\
$M_{*}\left[M_{\odot}\right]$ & $0.56 \pm 0.03$ & 0.570 & 0.664 \\
$\log g\left[\mathrm{~cm} / \mathrm{s}^{2}\right]$ & $7.91 \pm 0.07$ & - & 8.099 \\
$\log \left(L_{*} / L_{\odot}\right)$ & - & - & -1.14 \\
$\log \left(R_{*} / R_{\odot}\right)$ & - & - & -1.93 \\
$M_{\mathrm{He}, \text { total }}\left[M_{\odot}\right]$ & - & - & $3.6 \times 10^{-3}$ \\
\hline$M_{\text {env }}{ }^{*}$ & - & -2.80 & -1.63 \\
$M_{\mathrm{He}}{ }^{*}$ & - & -6.30 & -5.95 \\
$X_{\mathrm{O}}{ }^{* *}$ & - & $0.60-0.65$ & 0.65 \\
$q_{\mathrm{fm}}{ }^{* * *}$ & - & 0.36 & 0.46 \\
\hline
\end{tabular}

Notes. The second column corresponds to spectroscopic results (ØEA11), whereas the third and fourth columns present results from the asteroseismological study of BKØ11 and from the best-fit asteroseismological model of this work, respectively. ${ }^{(*)}$ Mass coordinate, defined as $\log \left(1-M_{r} / M_{*}\right)$ (see BKØ11). ${ }^{(*)}$ Central abundance by mass. ${ }^{(* * *)}$ Coordinate $M_{r}$ in units of $M_{*}$ (see BKØ11).

BKØ11 perform detailed period fits to KIC 8626021 by considering six parameters of their DB white dwarf models: $T_{\text {eff }}, M_{*}$, two parameters describing the $\mathrm{C}-\mathrm{O}$ core composition profiles $\left(X_{\mathrm{O}}, q_{\mathrm{fm}}\right)^{4}$, and two parameters that define the envelope structure $\left(M_{\mathrm{env}}, M_{\mathrm{He}}\right)^{5}$. They fix $M_{\mathrm{env}}$ and vary the remainder 5 parameters. In contrast, in our models we have only 2 free parameters: $T_{\text {eff }}$ and $M_{*}$, and the chemical structure at the core and envelope is kept fixed according to the predictions of the evolutionary computations. In order to compare the quality of our best fit with the results of BKØ11, we compute the Bayes Information Criterion (BIC; Koen \& Laney 2000):

$\mathrm{BIC}=N_{\mathrm{p}}\left(\frac{\log N}{N}\right)+\log \sigma^{2}$,

where $N_{\mathrm{p}}$ is the number of free parameters, and $N$ the number of observed periods. In our case, $N_{\mathrm{p}}=2$ (stellar mass and effective temperature). The smaller the value of $\mathrm{BIC}$, the better the quality of the fit. We obtain BIC $=0.85$, which is substantially larger than the BIC value of the best fit model of BKØ11 $(\mathrm{BIC}=-0.41)$. This means that our period fit is somewhat poorer than theirs. Notwithstanding this, our asteroseismological model still provides a very satisfactory fit to the periods of KIC 8626021.

The last column in Table 2 shows the rate of period change of the fitted pulsation modes. Our calculations predict all of the

${ }^{4} \mathrm{BK} \varnothing 11$ define $X_{\mathrm{O}}$ as the central $\mathrm{O}$ abundance and $q_{\mathrm{fm}}$ as the edge of the homogeneous $\mathrm{C}-\mathrm{O}$ core.

5 BKØ11 define $M_{\text {env }}$ as the mass coordinate of the base of the He layer, and $M_{\mathrm{He}}$ as the mass coordinate of the point where the He abundance raises to 1 . 
pulsation periods to increase with time $\left(\dot{\Pi}_{k}>0\right)$, in accordance with the decrease of the Brunt-Väisälä frequency in the core of the model induced by cooling. Note that at the effective temperature of KIC 8626021, cooling has the largest effect on $\dot{\Pi}_{k}$, while gravitational contraction, which should result in a decrease of periods with time, becomes negligible and no longer affects the pulsation periods. Until now, no measurement of $\dot{\Pi}$ in a DBV has been assessed, although important efforts to measure the rate of period change in at least one star at the blue edge (EC 20058-5234; Dalessio et al. 2010) are being currently carried out, and a possible determination of the rate of period change for other DBV star (PG 1351+489) has been reported (Redaelli et al. 2011).

The main features of our best-fit model are summarized in Table 3, where we also include the parameters of KIC 8626021 extracted from $\emptyset \mathrm{EA} 11$ and the seismological model derived by $\mathrm{BK} \varnothing 11$. In the table, the quantity $M_{\mathrm{He}, \text { total }}$ corresponds to the total content of $\mathrm{He}$ of the envelope of the model. In order to make the comparison easy, we include in the table the four parameters employed by $\mathrm{BK} \varnothing 11$ that define the chemical profiles at the core and envelope of their models. Note that BKØ11 do not specify the value of $M_{\mathrm{He} \text {,total }}$ of their best-fit model. The location of the best-fit model for KIC 8626021 both according to our study and that of BKØ11 in the $\log T_{\text {eff }}-\log g$ plane is shown in Fig. 1. Both asteroseismic studies lead to the conclusion that KIC 8626021 should be closer to the blue edge of the DBV instability strip than spectroscopy suggests.

\section{Discussion}

The results of our asteroseismological analysis point to a higher stellar mass of KIC 8626021 than predicted by spectroscopy. We arrive at such conclusion through both the period spacing and the individual periods exhibited by the star. Regarding the effective temperature, our work indicates a higher $T_{\text {eff }}$ than the spectroscopic measurement.

Our results also differ somewhat from those of the seismological analysis of BKØ11. Specifically, we obtain an asteroseismological model that is more massive and cooler than that of BKØ11. The fact that in both independent analysis a good match to the observed periods is found can be understood on the basis of the asymptotic behavior of $g$-mode pulsations, that predicts that a lower effective temperature is compensated by a higher mass. What is interesting and exciting is that, in spite of the substantial differences in the white dwarf modeling (in particular, the quite different composition profiles, that lead to significant differences in the pulsation periods) both analysis agree that KIC 8626021 is a hot DBV. This is in agreement with analyses based on the average period spacing and also with the fact that low period modes are present in KIC 8626021's pulsation spectrum, as is also observed for the hot DBV EC20058.

\section{Conclusions}

In this paper we have presented a detailed asteroseismic analysis of KIC 8626021, the first pulsating DB white dwarf star discovered by the Kepler mission, on the basis of the full evolutionary DB white-dwarf models presented in Althaus et al. (2009a) which were computed for a wide range of stellar masses and $\mathrm{He}$ envelopes. These DB white dwarf models are characterized by consistent chemical profiles for both the core and the envelope. These chemical profiles are the result of the computation of the full and complete evolution of the progenitor stars from the zero age main sequence, including the core $\mathrm{H}$ and $\mathrm{He}$ burning phases, the thermally pulsing asymptotic giant branch phase, the bornagain episode that is responsible for the $\mathrm{H}$ deficiency, and from time-dependent element diffusion predictions during the whitedwarf stage.

By considering the mean period spacing exhibited by the star, we found that KIC 8626021 should have a stellar mass in the range $0.60 \lesssim M_{*} / M_{\odot} \lesssim 0.87$, substantially larger than those derived by previous spectroscopic $\left(M_{*} \sim 0.56 M_{\odot}\right.$; ØEA11) and asteroseismic $\left(M_{*} \sim 0.57 M_{\odot}\right.$; BKØ11) studies. We also found that period-to-period fits point to an asteroseismological model with an effective temperature of $\sim 27300 \mathrm{~K}$, in strong conflict with the spectroscopic estimate $\left(T_{\text {eff }} \sim 24900 \mathrm{~K}\right)$. Our results are in agreement with the recent asteroseismic analysis of BKØ11 on KIC 8626021, in particular regarding its effective temperature. In fact, these authors conclude that KIC 8626021 must be a hot DBV with $T_{\text {eff }} \sim 29200 \mathrm{~K}$. It would be interesting to see what a spectroscopic analysis based on higher signalto-noise spectra will tell about the surface gravity and effective temperature of the star.

If KIC 8626021 is a hot DBV, as first found by BKØ11 and confirmed now by our results, then further monitoring of KIC 8626021 with Kepler in the next years probably will allow a measurement of $\dot{\Pi}$, which in turn could open the possibility to constrain the plasmon neutrino emission rate (Winget et al. 2004; BKØ11). This endeavour is evidently dependent on the models, which can always be improved. Uncertainties in the models can also be assessed (Bischoff-Kim et al. 2008).

Acknowledgements. This work was supported by AGENCIA: Programa de Modernización Tecnológica BID 1728/OC-AR, and by PIP 2008-00940 from CONICET. This research has made use of NASA's Astrophysics Data System.

\section{References}

Althaus, L. G., \& Córsico, A. H. 2004, A\&A, 417, 1115

Althaus, L. G., Serenelli, A. M., Panei, J. A., et al. 2005, A\&A, 435, 631

Althaus, L. G., Panei, J. A., Miller Bertolami, M. M., et al. 2009a, ApJ, 704, 1605

Althaus, L. G., García-Berro, E., Córsico, A. H., Miller Bertolami, M. M., \& Romero, A. D. 2009b, ApJ, 693, L23

Althaus, L. G., Panei, J. A., Romero, A. D., et al. 2009c, A\&A, 502, 207

Althaus, L. G., Córsico, A. H., Isern, J., \& García-Berro, E. 2010, A\&ARv, 18, 471

Angulo, C., Arnould, M., Rayet, M., et al. 1999, Nucl. Phys. A, 656, 3

Beauchamp, A., Wesemael, F., Bergeron, P., et al. 1999, ApJ, 516, 887

Bischoff-Kim, A., \& Østensen, R. H. 2011, ApJ, 742, L16 (BKØ11)

Bischoff-Kim, A., Montgomery, M. H., \& Winget, D. E. 2008, ApJ, 675, 1512

Bradley, P. A., \& Winget, D. E. 1994, ApJ, 421, 236

Brickhill, A. J. 1991, MNRAS, 251, 673

Cassisi, S., Potekhin, A. Y., Pietrinferni, A., Catelan, M., \& Salaris, M. 2007, ApJ, 661, 1094

Caughlan, G. R., \& Fowler, W. A. 1988, Atomic Data and Nuclear Data Tables, 40, 283

Castanheira, B. G., Kepler, S. O., Kleinman, S. J., Nitta, A., \& Fraga, L. 2010, MNRAS, 405, 2561

Córsico, A. H., \& Althaus, L. G. 2006, A\&A, 454, 863

Córsico, A. H., Althaus, L. G., \& Miller Bertolami, M. M. 2006, A\&A, 458, 259

Córsico, A. H., Althaus, L. G., Miller Bertolami, M. M., \& Werner, K. 2007a, A\&A, 461, 1095

Córsico, A. H., Miller Bertolami, M. M., Althaus, L. G., Vauclair, G., \& Werner, K. 2007b, A\&A, 475, 619

Córsico, A. H., Althaus, L. G., Kepler, S. O., Costa, J. E. S., \& Miller Bertolami, M. M. 2008, A\&A, 478, 869

Córsico, A. H., Althaus, L. G., Miller Bertolami, M. M., \& García-Berro, E. 2009, A\&A, 499, 257

Dalessio, J., Provencal, J. L., Sullivan, D. J., \& Shipman, H. L. 2010, AIP Conf. Ser., 1273, 536

Dehner, B. T., \& Kawaler, S. D. 1995, ApJ, 445, L141

Gautschy, A., \& Althaus, L. G. 2002, A\&A, 382, 141

Goldreich, P., \& Wu, Y. 1999, ApJ, 511, 904 
Haft, M., Raffelt, G., \& Weiss, A. 1994, ApJ, 425, 222

Hermes, J. J., Mullally, F., Østensen, R. H., et al. 2011, ApJ, 741, L16

Iglesias, C. A., \& Rogers, F. J. 1996, ApJ, 464, 943

Isern, J., García-Berro, E., Althaus, L. G., \& Córsico, A. H. 2010, A\&A, 512, A86

Itoh, N., Hayashi, H., Nishikawa, A., \& Kohyama, Y. 1996, ApJS, 102, 411

Kilkenny, D., O’Donoghue, D., Crause, L. A., Hambly, N., \& MacGillivray, H. 2009, MNRAS, 397, 453

Koen, C., \& Laney, D. 2000, MNRAS, 311, 636

Magni, G., \& Mazzitelli, I. 1979, A\&A, 72, 134

Miller Bertolami, M. M., \& Althaus, L. G. 2006, A\&A, 454, 845

Miller Bertolami, M. M., Althaus, L. G., Unglaub, K., \& Weiss, A. 2008, A\&A, 491,253

Nitta, A., Kleinman, S. J., Krzesinski, J., et al. 2009, ApJ, 690, 560
Østensen, R. H., Bloemen, S., Vučković, M., et al. 2011, ApJ, 736, L39 (ØEA11) Redaelli, M., Kepler, S. O., Costa, J. E. S., et al. 2011, MNRAS, 415, 1220

Romero, A. D., Córsico, A. H., Althaus, L. G., et al. 2012, MNRAS, 420, 1462

Segretain, L., Chabrier, G., Hernanz, M., et al. 1994, ApJ, 434, 641

Tassoul, M., Fontaine, G., \& Winget, D. E. 1990, ApJS, 72, 335

Winget, D. E., \& Kepler, S. O. 2008, ARA\&A, 46, 157

Winget, D. E., van Horn, H. M., Tassoul, M., et al. 1982a, ApJ, 252, L65

Winget, D. E., Robinson, E. L., Nather, R. D., \& Fontaine, G. 1982b, ApJ, 262, L11

Winget, D. E., van Horn, H. M., Tassoul, M., Hansen, C. J., \& Fontaine, G. 1983, ApJ, 268, L33

Winget, D. E., Sullivan, D. J., Metcalfe, T. S., Kawaler, S. D., \& Montgomery, M. H. 2004, ApJ, 602, L109 